\title{
ON THE ALEXANDER POLYNOMIAL OF ALTERNATING ALGEBRAIC KNOTS
}

\author{
KUNIO MURASUGI
}

(Received 13 December 1983; revised 28 May 1984)

Communicated by J. H. Rubinstein

\begin{abstract}
A conjecture of Fox about the coefficients of the Alexander polynomial of an alternating knot is proved for alternating algebraic (or arborescent) knots, which include two-bridge knots.

1980 Mathematics subject classification (Amer. Math. Soc.): 57 M 25.
\end{abstract}

\section{Introduction}

An integer polynomial $f(t)=\sum_{n=0}^{m} c_{n} t^{n}$ will be called trapezoidal if

(1) all the coefficients of $f(t)$ are nonzero and have the same sign,

(2) $t^{m} f(1 / t)=f(t)$,

(3) $\left|c_{0}\right| \leqslant\left|c_{1}\right| \leqslant \cdots \leqslant\left|c_{k}\right|, k=[m / 2]$ and

(4) whenever $c_{i}=c_{i+1}, c_{i}=c_{j}, i \leqslant j \leqslant[m / 2]$.

We call $f(t)$ positive or negative according to whether $c_{0}>0$ or $c_{0}<0$.

If $\Delta(t)$ is the Alexander polynomial of an alternating knot, then $\Delta(-t)$ is trapezoidal in all cases thus far examined. This naturally leads to the following conjecture [4].

CONJECTURE. Let $\Delta(t)$ denote the Alexander polynomial of an alternating knot in $S^{3}$. Then $\Delta(-t)$ is trapezoidal.

(C) 1985 Australian Mathematical Society $0263-6115 / 85$ \$A2.00 + 0.00 
The conjecture has been proved only for 2-bridge knots [5] and a few other special types of alternating knots like alternating prezel knots [10]. Proofs are, in principle, based upon the actual computation of the Alexander polynomials.

The purpose of this paper is to prove the conjecture for a large family of alternating algebraic knots which includes, in particular, 2-bridge knots (see Theorem 5.1). To define our family of alternating algebraic knots or links, first, we will give a sufficient condition for $l$ to be alternating in terms of the associated weighted tree (see Section 4). The conjecture will be proved for alternating links that satisfy this sufficient condition by a simple inductive argument. To avoid the involvement of the link matrices in the proof, we will use the Conway polynomials. The proof is, therefore, probably more comprehensible but not simpler, than the use of the link matrices.

\section{Preliminaries}

Throughout this paper, a link means the union of $n(\geqslant 1)$ disjoint 1-sphere $S^{1}$ in $S^{3}$, but a knot is always to mean a link of one component. All links are oriented.

By the reduced link polynomial of $l$, denoted $\Delta_{l}(t)$, is meant the reduced Alexander polynomial of $l$ and hence, if $l$ is a knot, $\Delta_{l}(t)$ is the ordinary Alexander polynomial.

Now the following easy proposition will be used frequently in this paper. The proof will be omitted, however, since it is elementary, but tedious.

Proposition 2.1. Let $f(t)=\sum_{i=0}^{m} c_{i} t^{i}$ and $g(t)=\sum_{j=0}^{n} d_{j} t^{j}$ be positive trapezoidal polynomials. Then

(1) $f(t) g(t)$ is trapezoidal,

(2) if $m=n+2 k, 0 \leqslant k \leqslant 1$, then $f(t)+t^{k} g(t)$ is trapezoidal.

Now let $l$ be an algebraic link (or an arborescent link) in $S^{3}$ [2]; $l$ is associated with a weighted (finite) tree $T$. In this paper, we only consider finite graphs, and therefore, a graph is always to mean a finite graph. Let $w$ be a weight function.

Definition 2.1. A vertex $a_{i}$ of $T$ is called positive (or negative) if $w\left(a_{i}\right)>0$ (or $\left.w\left(a_{i}\right)<0\right) ; T$ is called positive (or negative) if all the vertices are positive (or negative). $T$ is called even if $w\left(a_{i}\right) \equiv 0(\bmod 2)$ for all $a_{i}$. In particular, $T$ is called a 0-tree if $w\left(a_{i}\right)=0$ for all $a_{i}$. Let $v\left(a_{i}\right)$ denote the valency of $a_{i}$, i.e. the number of edges incident with $a_{i}$. A vertex $a_{i}$ is called a stump if $v\left(a_{i}\right)=1$, and $a_{i}$ is called a twig if $v\left(a_{i}\right)=2$. T is called excessive if $\left|w\left(a_{i}\right)\right| \geqslant \max \left\{v\left(a_{i}\right), 2\right\}$ for all $a_{i}$.

An algebraic link $l$ is the boundary of a surface $F$ constructed by plumbing as specified by a weighted tree $T ; l$ will be denoted by $l(T)$ and $F$ by $F(T)$. 
EXAMPLE 1.

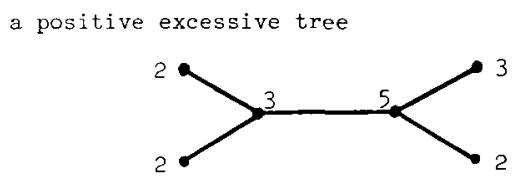

an excessive tree
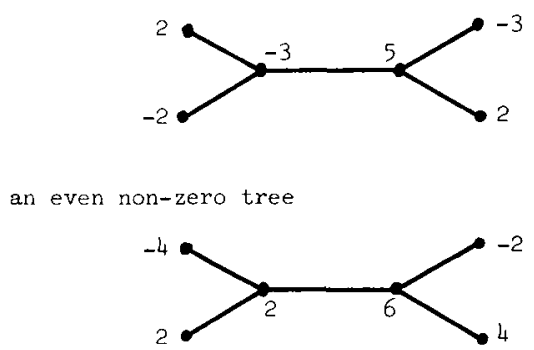

FIGURE 1

In this paper, a disjoint union of trees (usually called a forest) will also be called a tree. Therefore, a tree may be disconnected.

\section{DEFINITION 2.2 .}

(1) A vertex-graph is a graph with just one vertex and no edge.

(2) An edge-graph is a tree with just one edge and two stumps.

(3) An arc-tree is a connected tree having just two stumps.

(4) The complement of a subtree $H$ of a tree $T$, denoted by $\hat{T}(H)$, is the tree obtained from $T$ by removing all vertices of $H$ and all edges of $T$ incident with at least one vertex in $H$. Therefore, $\hat{T}(H)$ is, in general, a collection of trees.

(5) A root is a vertex with which at least two stumps are incident.

A reduction on $T$ that shrinks a 2 -edge arc-tree to a vertex will be called a contraction. See Figure 2.

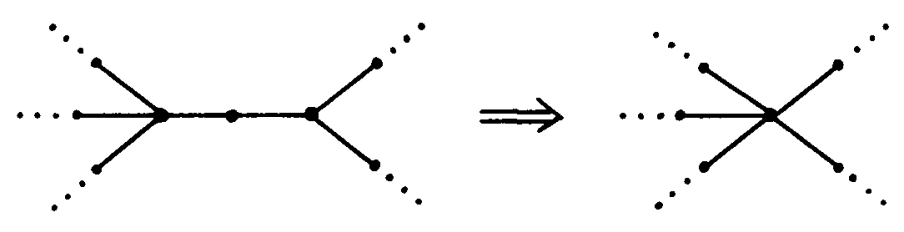

Figure 2

Sometimes, a contraction will be called an elimination of a twig. 
It is easy to see that if a connected tree $T$ does not have a root, then $T$ has a twig unless $T$ is an edge-graph or a vertex-graph.

Proposition 2.2. By a finite number of applications of contractions, any connected tree can be reduced to a graph containing at least one root, or an edge-graph, or a vertex-graph.

The proof is obvious.

Given an even tree $T$, we are able to determine whether or not the link $l(T)$ is a knot.

Proposition 2.3. For an even tree $T, l(T)$ is a knot if and only if $T$ is reduced to an edge-graph by a finite number of applications of contraction.

Proof. Since we are only concerned about the number of components of $l(T)$, we may assume that $T$ is a 0 -tree. Since a contraction then does not change the link type, $T$ may be assumed to be one of the three graphs listed in Proposition 2.2. Obviously, $l(T)$ is a knot if and only if $T$ can be reduced to an edge-graph.

Corollary 2.4. Let $T$ be a 0 -tree. Then $\Delta_{l(T)}(t)$ equals 1 or 0 according as $l(T)$ is a knot or not.

Proof. $l(T)$ is either an unknotted knot or a split link, and hence $\Delta_{l(T)}(t)$ equals 1 or 0 .

\section{Graphs and links}

Let $G$ be a finite planar graph without loops or stumps; $G$ divides a plane into finitely many domains $D_{0}, D_{1}, \ldots, D_{m}$, say. Take a point $P_{i}$ from each domain $D_{i}$ and join $P_{i}, P_{j}$ whenever $\partial D_{i} \cap \partial D_{j} \neq \varnothing$, by simple arcs, each of which intersects exactly one point in the common edge. Therefore if $\partial D_{i} \cap \partial D_{j}$ consists of $k$ edges $\sigma_{1}, \sigma_{2}, \ldots, \sigma_{k}$, we join $P_{i}$ and $P_{j}$ by $k$ simple arcs $\tau_{1}, \tau_{2}, \ldots, \tau_{k}$ in such a way that $\sigma_{p} \cap \tau_{q} \neq \varnothing$ if and only if $p=q$. A new planar graph $G^{*}$ thus obtained is called the dual graph of $G$. Note that a vertex $P_{j}$ of $G^{*}$ corresponds to a domain $D_{j}$.

A graph is called a directed graph if + or - is assigned to each edge. When $G$ is a directed graph, the dual graph $G^{*}$ is also directed by putting + on $\tau_{i}$ if and only if $\sigma_{i}$ is a positive edge. 
Definition 3.1. A planar graph $G$ is called collapsible if the collection of the domains $\left\{D_{0}, D_{1}, \ldots, D_{m}\right\}$ can be renumbered (if necessary) so that

(1) $D_{0}$ is the unbounded domain,

(2) for $i=1,2, \ldots, m-1, D_{1} \cup \cdots \cup D_{i}$ and $D_{i+1}$ have at most one edge in common.

EXAMPLE 2.

collapsible

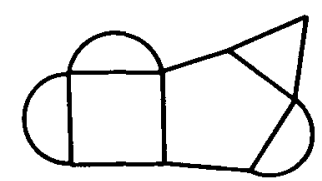

non-collapsible

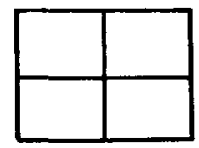

Figure 3

Proposition 3.1. A planar graph (without loops or stumps) is collapsible if and only if there is a vertex $v$ in the dual graph $G^{*}$ of $G$ such that the complement of $v$ in $G^{*}$ is a tree.

Proof. Suppose that $G$ is collapsible. Then the vertex corresponding to the unbounded domain is what we sought.

The converse is obvious. Simply renumber the domains $D_{0}, D_{1}, \ldots, D_{m}$ along the tree.

Now let $T$ be a weighted tree on a plane. We assume $\left|w\left(a_{i}\right)\right| \geqslant 2$ if $a_{i}$ is a stump. Assign + or - to each edge of $T$ arbitrarily. Then take a point $a_{0}$, not on $T$, from the plane. Join $a_{0}$ and each $a_{i}(i \neq 0)$ by disjoint simple arcs and assign + or - on each new arc in such a way that the number of positive arcs minus the number of negative arcs incident with $a_{i}$ is equal to $w\left(a_{i}\right)$. Thus we obtain a directed planar graph $G$.

EXAMPLE 3.

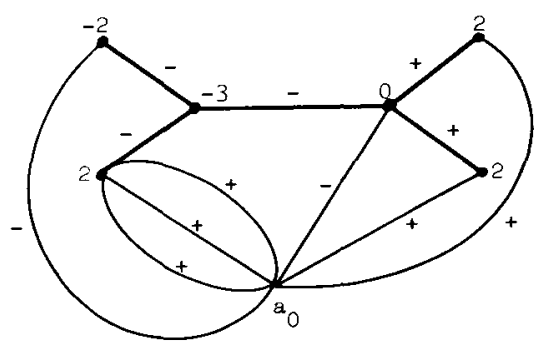

Figure 4 
Consider the directed dual graph $G^{*}$ of $G$. Proposition 3.1 then implies

Proposition 3.2. $G^{*}$ is collapsible.

From this directed planar graph $G^{*}$, we can construct a link $l$ as follows. First we replace each vertex by a small disk and each edge by a band in the plane connecting the two corresponding disks. If each band is then twisted in the positive or negative direction according as the corresponding edge is positive or negative without causing any "complication" in the resulting surface $F$, this surface $F$ will have spine $G^{*}$. (See Figure 5 below.) The boundary of the (not necessarily orientable) surface $F$ is the link $l$ we sought.

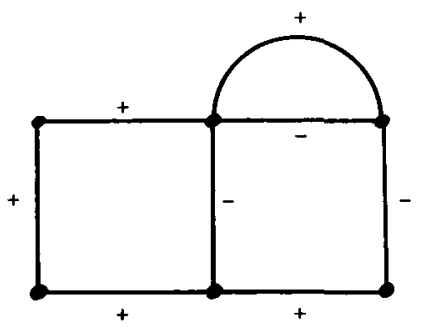

(a)

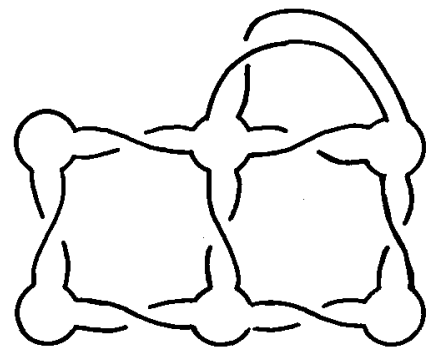

(b)

Figure 5

Proposition 3.3. $l$ is ambient isotopic to the algebraic link associated with the weighted tree $T$.

Proof. Let $F$ be the (not necessarily orientable) surface constructed from $G^{*}$ and let $\alpha$ be a band "surrounding" a stump $a_{1}$ of $T$ (Figure 6(a)). Slide $\alpha$ along a line $A B$ (Figure 6(b)). Then we can say that $F$ is obtained from $\alpha$ and the rest of the surface $F_{0}$ by plumbing. Since $F_{0}$ is associated with a tree $\hat{T}\left(a_{1}\right)$, the induction can be applied.

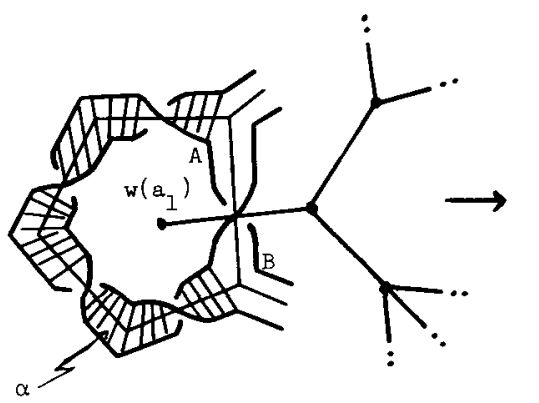

(a)

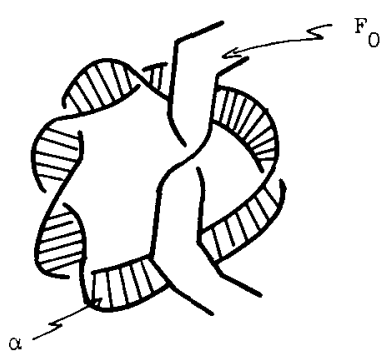

(b)

Figure 6 
Proposition 3.3 shows that given a weighted tree $T$, two collapsible planar graphs constructed from $T$ define ambient isotopic links.

\section{A criterion for alternating links}

If a weighted tree $T$ is positive (or negative) and is excessive, then a collapsible planar graph $G^{*}$ constructed in the previous section can be chosen so that all the edges of $G^{*}$ are positive (or negative). Therefore, the link obtained from $G^{*}$ is obviously alternating, that is, we have

Proposition 4.1. If $T$ is a positive (or negative) excessive tree, then the algebraic link $l(T)$ is alternating.

For a positive but not excessive tree $T, l(T)$ may not be alternating.

EXAMPLE 4.

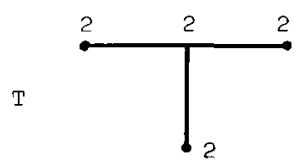

Figure 7

Now consider an arbitrary weighted tree $T$.

Let $A(T)$ be the set of those edges in $T$ which join positive vertices and negative vertices. If all the edges in $A(T)$ are removed from $T, T$ will split into finitely many subtrees $T_{1}, T_{2}, \ldots, T_{k}$, each of which is either strictly positive or negative. A collection $\left\{T_{1}, T_{2}, \ldots, T_{k}\right\}$ will be called a uniform decomposition of $T$.

\section{EXAMPLE 5.}
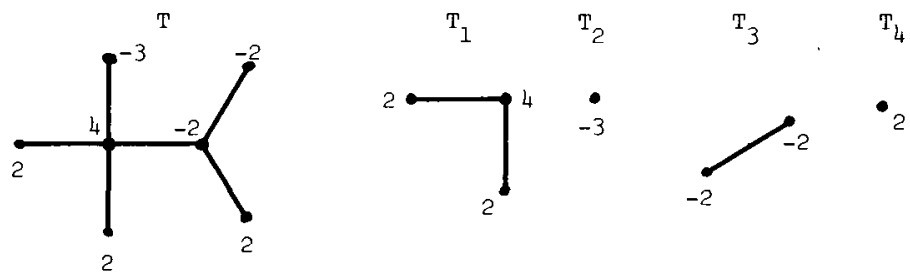

FIGURE 8 
Proposition 4.2. Let $\left\{T_{1}, T_{2}, \ldots, T_{k}\right\}$ be the uniform decomposition of a tree $T$. If each of $T_{1}, \ldots, T_{k}$ is excessive, then the link $l(T)$ is alternating.

Proof. Let $V$ be the set of those vertices of $T$ which are incident with some edges in $A(T)$. Let $G^{*}$ be the collapsible planar graph constructed in Section 3 . Since each $T_{i}$ is positive or negative, $G^{*}$ can be chosen so that all edges of the boundary of the domain corresponding to a vertex in $\hat{T}(V)$ have the same sign as that of $T_{i}$. Then slide a band along the edge dual to an edge in $A(T)$, as we did in the proof of Proposition 3.3. The new surface is obtained by plumbing the surface $F\left(T_{1}\right), F\left(T_{2}\right), \ldots, F\left(T_{k}\right)$, and it bounds $l(T)$. When $F\left(T_{i}\right)$ and $F\left(T_{j}\right)$ are plumbed, $T_{i}$ and $T_{j}$ have opposite signs and hence, the boundary of $F(T)$ is alternating. (Compare the construction of Seifert surfaces for alternating links [7].)

COROLlaRY 4.3. A 2-bridge link is alternating.

Proof. A 2-bridge link is associated with a non-zero even arc-tree $T$. Therefore, each component of the uniform decomposition of $T$ is excessive. The corollary follows from Proposition 4.2.

\section{Statement of main theorem}

Now we begin to study the reduced link polynomials of algebraic links.

Suppose we are given a weighted tree $T$. If the surface $F(T)$ is orientable, the orientation of $F(T)$ induces an orientation of the link $l(T)$ and hence $F(T)$ is a Seifert surface of $l(T)$. This makes the computation of the link polynomials much easier.

Since $F(T)$ is orientable if and only if $T$ is even, we consider hereafter only even trees. We assume, further, the orientation of $l(T)$ is induced from that of $F(T)$, whenever $F(T)$ is orientable. Then the main theorem of this paper is

THEOREM 5.1. Let $T$ be an even tree and let $\Delta_{l}(t)$ be the reduced link polynomial of the algebraic link $l(T)$ associated with $T$. Let $\left\{T_{1}, T_{2}, \ldots, T_{k}\right\}$ be the uniform decomposition of $T$. If each $T_{i}$ is excessive, then $l(T)$ is alternating and $\Delta_{l}(-t)$ is trapezoidal.

The first half of Theorem 5.1 is included in Proposition 4.2. The later half will be proved in Sections 6-8 by induction. 


\section{Conway polynomial}

Let $l$ be an oriented link and let $\nabla_{l}(z)$ denote the Conway polynomial of $l$; $\nabla_{l}(z)$ is an integer polynomial on $z$ and it is recursively computable by using a few axioms [3, 6]. In particular, it satisfies the following axiom.

(6.1) If $l, l^{\prime}$ and $l_{0}$ are links differing at one crossing as indicated below, then $\nabla_{l}(z)=\nabla_{l^{\prime}}(z)+z \nabla_{l_{0}}(z)$.

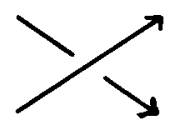

$\ell$

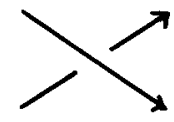

$\ell^{\prime}$

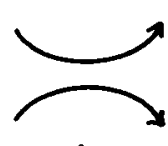

$\ell_{0}$

FIGURE 9

This axiom (6.1) is slightly different from that given in [6]. However, it defines the same polynomial.

Now let $T$ be a connected even tree. Since $F(T)$ is orientable and its orientation induces the orientation of $l(T)$, the Conway polynomial of $l(T)$ is uniquely determined, and is denoted by $\nabla(z ; T)$.

For an even tree $T$ with $k$ connected components $T_{1}, T_{2}, \ldots, T_{k}, \nabla(z ; T)$ is defined as $\nabla(z ; T)=\prod_{i=1}^{k} \nabla\left(z ; T_{i}\right)$. It should be noted that for a connected tree $T, \nabla(z ; T)$ may be 0 , but for a disconnected tree, $\nabla(z ; T)$ may not be 0 .

The purpose of this section is to prove a simple reduction formula (6.3) below.

Let $a_{i}$ be a vertex of $T$. Suppose $q$ edges $e_{1}, e_{2}, \ldots, e_{q}$ are incident with $a_{i}$. We define two weighted trees $U_{1}$ and $U_{2}$ as follows.

$U_{1}$ is a tree obtained from $T$ by removing $p$ edges $e_{1}, e_{2}, \ldots, e_{p},(0 \leqslant p \leqslant q)$, while $U_{2}$ is a subtree of $T$ obtained by removing the remaining $q-p$ edges $e_{p+1}, \ldots, e_{q}$. The weight functions $w_{j}$ for $U_{j}(j=1,2)$ will be defined by means of the weight function $w$ of $T$ :

(1) if $r \neq i, w_{j}\left(a_{r}\right)=w\left(a_{r}\right), j=1,2$,

(2) $w_{1}\left(a_{i}\right)$ and $w_{2}\left(a_{i}\right)$ are even, and

(3) $w_{1}\left(a_{i}\right)+w_{2}\left(a_{i}\right)=w\left(a_{i}\right)$.

We say $T$ splits into $U_{1}$ and $U_{2}$. (See Example 6 in Section 7.) Then we have

Proposition 6.1.

$$
\nabla(z ; T)=\nabla\left(z ; U_{1}\right)+\nabla\left(z ; U_{2}\right)
$$

Proof. In the following proof, we will distinguish between knots and links (of more than one component). 
Now first we should note that the complements $\hat{T}\left(a_{i}\right), \hat{U}\left(a_{i}\right)$ and $\hat{U}_{2}\left(a_{i}\right)$ are identical and consist of $q$ connected trees $T_{1}, T_{2}, \ldots, T_{q}$. Let $a_{j_{k}}, k=1,2, \ldots, q$, be the vertex which is connected to $a_{i}$ by the edge $e_{k} ; a_{j_{k}}$ belongs to $T_{k}$.

Case 1. all $T, U_{1}$ and $U_{2}$ are 0 -trees.

Since (6.3) is trivially true when $p=0$ or $p=q$, we assume $0<p<q$ and hence, $q \geqslant 2$.

Since, for a 0 -tree $T, \nabla(z ; T)$ is 1 or 0 according as $l(T)$ is a knot or a link, it suffices to prove

(1) all $l(T), l\left(U_{1}\right)$ and $l\left(U_{2}\right)$ are links, or

(2) if $l(T)$ is a knot, then either $l\left(U_{1}\right)$ or $l\left(U_{2}\right)$ is a knot, but not both.

We will prove (6.4) by induction on $\mu(T)$, the number of edges in $T$.

The initial case is $\mu(T)=q$. Then $a_{i}$ is a root in $T$, and hence $l(T)$ is a link. On the other hand, each of $U_{1}$ and $U_{2}$ contains at least one vertex-graph, and hence both $l\left(U_{1}\right)$ and $l\left(U_{2}\right)$ are links.

Now assume that (6.4) holds for any tree $T^{\prime}$ with $\mu\left(T^{\prime}\right)<\mu(T)$.

If $T$ has a root, then $l(T), l\left(U_{1}\right)$ and $l\left(U_{2}\right)$ are all links. In fact, if some vertex $a_{m}\left(\neq a_{i}\right)$ is a root, then $a_{m}$ is also a root in $U_{1}$ and $U_{2}$. Therefore, $l(T), l\left(U_{1}\right)$ and $l\left(U_{2}\right)$ are all links. If $a_{i}$ is a root in $T$, then at least two of $q$ vertices $a_{j_{1}}, a_{j_{2}}, \ldots, a_{j_{q}}$ are stumps in $T$. Let $a_{j_{s}}$ and $a_{j_{w}}$ be stumps in $T$. If both $e_{s}$ and $e_{w}$ belong to $U_{1}$, then $a_{i}$ is a root in $U_{1}$, while $U_{2}$ contains two vertex-graphs $\left\{a_{j_{s}}\right\},\left\{a_{j_{w}}\right\}$. If $e_{s} \in U_{1}$, but $e_{w} \in U_{2}$, then $\left\{a_{j_{w}}\right\}$ and $\left\{a_{j_{s}}\right\}$ are vertex-graphs in $U_{1}$ and $U_{2}$, respectively. Therefore $l(T), l\left(U_{1}\right)$ and $l\left(U_{2}\right)$ are all links.

Now suppose that $T$ has no roots. Since $T$ is neither an edge-graph nor a vertex-graph, $T$ must have a twig, which may be eliminated by a contraction. Apply the induction hypothesis.

Case 2. general case.

Let $\lambda(T)$ be the number of non-zero vertices in $T$.

For $\lambda(T)=0$, we are done in Case 1 .

Suppose (6.3) holds for any tree $T^{\prime}$ with $\lambda\left(T^{\prime}\right)<\lambda(T)$. We say that the tree $T_{0}$ is obtained from $T$ by defining a new weight $w\left(a_{j}\right)=c$, if $T$ and $T_{0}$ have the same underlying tree, but weight functions differ (possibly) at only one vertex $a_{j}$.

LEMMA 6.2. Denote by $V(n)$ the vertex-graph with weight $n$. Let $T$ be a weighted tree with $w\left(a_{i}\right)=2 b>0$. Let $T_{0}$ be the tree obtained from $T$ by defining $a$ new weight $w\left(a_{i}\right)=0$. Then

$$
\nabla(z ; T)=\nabla\left(z ; T_{0}\right)+\nabla\left(z ;\left\{\hat{T}\left(a_{i}\right), V(2 b)\right\}\right) .
$$


Proof. If we apply Axiom (6.1) at a crossing associated with a vertex $a_{i}$, we obtain $\nabla(z ; T)=\nabla\left(z ; T_{1}\right)+z \nabla\left(z ; \hat{T}\left(a_{i}\right)\right)$, where $T_{1}$ is the tree obtained from $T$ by defining a new weight $w\left(a_{i}\right)=2(b-1)$. Since $z=\nabla(z ; V(2))$, we can write $z \nabla\left(z ; \hat{T}\left(a_{i}\right)\right)=\nabla\left(z ;\left\{\hat{T}\left(a_{i}\right), V(2)\right\}\right)$. By repeating this $b$ times, we obtain the lemma.

Now we return to a proof of Proposition 6.1.

Suppose $w\left(a_{i}\right)=2 b \neq 0$ and $b>0$ (for the case $b<0$, a similar proof is valid). By Lemma 6.2, we have

$$
\nabla(z ; T)=\nabla\left(z ; T_{0}\right)+\nabla\left(z ;\left\{\hat{T}\left(a_{i}\right), V(2 b)\right\}\right) .
$$

Since $\lambda\left(T_{0}\right)=\lambda(T)-1<\lambda(T)$, it follows by the induction assumption that $\nabla\left(z ; T_{0}\right)=\nabla\left(z ; U_{1}^{\prime}\right)+\nabla\left(z ; U_{2}^{\prime}\right)$, where $U_{j}^{\prime}(j=1,2)$ are trees obtained from $U_{j}$ by defining new weights $w\left(a_{i}\right)=0(j=1,2)$. Write $w_{1}\left(a_{i}\right)=2 b^{\prime}$ and $w_{2}\left(a_{i}\right)=$ $2 b^{\prime \prime}$. Since $2 b=2 b^{\prime}+2 b^{\prime \prime}$, we have

$$
\nabla\left(z ;\left\{\hat{T}\left(a_{i}\right), V(2 b)\right\}\right)=\nabla\left(z ;\left\{\hat{T}\left(a_{i}\right), V\left(2 b^{\prime}\right)\right\}\right)+\nabla\left(z ;\left\{\hat{T}\left(a_{i}\right), V\left(2 b^{\prime \prime}\right)\right\}\right) .
$$

Lemma 6.2 also implies

$$
\begin{aligned}
& \nabla\left(z ; U_{1}\right)=\nabla\left(z ; U_{1}^{\prime}\right)+\nabla\left(z ;\left\{\hat{T}\left(a_{i}\right), V\left(2 b^{\prime}\right)\right\}\right) \quad \text { and } \\
& \nabla\left(z ; U_{2}\right)=\nabla\left(z ; U_{2}^{\prime}\right)+\nabla\left(z ;\left\{\hat{T}\left(a_{i}\right), V\left(2 b^{\prime \prime}\right)\right\}\right) .
\end{aligned}
$$

Combining (6.5)-(6.7), we obtain (6.3).

Finally, suppose $w\left(a_{i}\right)=0$, but $w\left(a_{r}\right)=2 c \neq 0$ for some vertex $a_{r}$. We may assume without loss of generality that $a_{r}$ belongs to $U_{1}$.

Let $T_{0}$ and $U_{j}^{\prime}(j=1,2)$ be trees obtained from $T$ and $U_{j}$, respectively, by defining new weights $w\left(a_{r}\right)=0$. Then it follows from Lemma 6.2 that

$$
\nabla(z ; T)=\nabla\left(z ; T_{0}\right)+\nabla\left(z ;\left\{\hat{T}\left(a_{r}\right), V(2 c)\right\}\right) .
$$

Since $\lambda\left(T_{0}\right)<\lambda(T)$ and $\lambda\left(\hat{T}\left(a_{r}\right)\right)<\lambda(T)$, we obtain, by the induction assumption,

$$
\begin{aligned}
& \nabla\left(z ; T_{0}\right)=\nabla\left(z ; U_{1}^{\prime}\right)+\nabla\left(z ; U_{2}^{\prime}\right), \quad \text { and } \\
& \nabla\left(z ;\left\{\hat{T}\left(a_{r}\right), V(2 c)\right\}\right)=\nabla\left(z ; \hat{U}_{1}\left(a_{r}\right)\right)+\nabla\left(z ; \hat{U}_{2}\left(a_{r}\right)\right) .
\end{aligned}
$$

Applying Lemma 6.2 to $U_{1}$ and $U_{2}$, we obtain

$$
\nabla\left(z ; U_{j}\right)=\nabla\left(z ; U_{j}^{\prime}\right)+\nabla\left(z ; \hat{U}_{j}\left(a_{r}\right)\right), \quad j=1,2 .
$$

Equation (6.3) now follows from (6.8)-(6.10). This proves Proposition 6.1. 


\section{Proof of main theorem (I)}

Let $\nabla(z ; T)=\sum_{i=0}^{m} a_{i} z^{i}, a_{m} \neq 0$, be the Conway polynomial of a link $l(T)$. Since $t^{m} \nabla(t-1 / t ; T)$ is an integer polynomial on $t^{2}$, we can define an integer polynomial $\Delta(t ; T)$ so that $\Delta\left(t^{2} ; T\right)=t^{m} \nabla(t-1 / t ; T)$. Then it is known [3,6] that $\Delta(t ; T)$ is equal to the reduced link polynomial $\Delta_{l}(t)$ of $l(T)$ up to $\pm t^{k}$. The degree of $\Delta(t ; T)$ is equal to the degree of $\nabla(z ; T)$. We will denote by sign $\Delta(t ; T)$ the sign of the leading coefficient of $\Delta(t ; T)$ (and hence, of $\nabla(z ; T)$ ).

Now, although (6.3) holds for any even tree, it is particularly useful for alternating algebraic links. In fact, for an even excessive tree $T$, the surface $F(T)$ is orientable and $F(T)$ is a Seifert surface of $l(T)$ with minimum genus, and hence, the Betti number of $H_{1}(F(T), Z)$ is exactly the number of vertices in $T$. Therefore, we have

Proposition 7.1 [7]. Let $T$ be an even positive excessive tree and $n$ the number of vertices in $T$. Then

(1) $n=\operatorname{deg} \nabla(z ; T)=\operatorname{deg} \Delta(t ; T)$,

(2) $\Delta(0 ; T) \neq 0$,

(3) $\operatorname{sign} \Delta(-t, T)=(-1)^{n}$,

(4) $(-1)^{n} \Delta(-t ; T)$ is a positive polynomial,

(5) if both $U_{1}$ and $U_{2}$ (used in (6.3)) are positive excessive even trees, then

$$
\Delta(t ; T)=\Delta\left(t ; U_{1}\right)+\Delta\left(t ; U_{2}\right)
$$

Now, using (6.3) or (7.1), the computation of the reduced link polynomial is reduced to those of simpler links. Since the simplest algebraic links are 2-bridge links, first we consider these links.

A 2-bridge link $l$ is associated with an even arc-tree $T$ below.

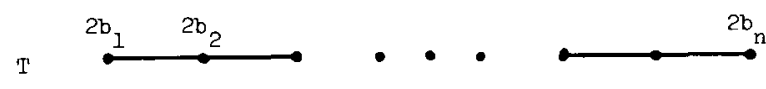

FIGURE 10

Proposition 7.2. If all $b_{i}$ are positive, then $\Delta_{l}(-t)$ is trapezoidal.

Proof. If $n=1$, then $\Delta_{l}(-t)=b_{1}(1+t)$, which is trapezoidal. If all $b_{i}=1$, then $l$ is a torus link of type $(n, 2)$ and $\Delta_{l}(-t)=1+t+t^{2}+\cdots+t^{n-1}$, which is trapezoidal. 
Now suppose that some $b_{i}$ is greater than 1 . Then split $T$ into two subtrees $U_{1}$ and $U_{2}$ as is shown in Figure 11 below.

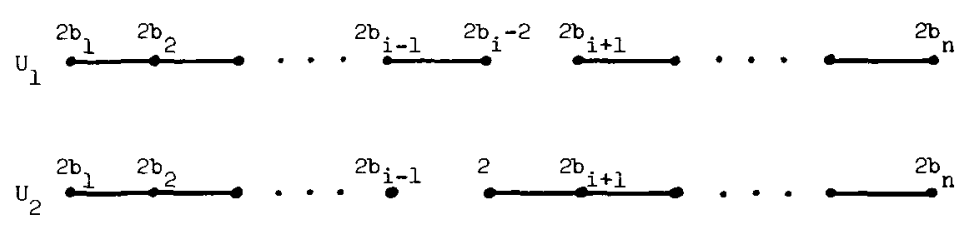

FIGURE 11

Since $U_{1}$ and $U_{2}$ are even excessive, (7.1) yields $\Delta(t ; T)=\Delta\left(t ; U_{1}\right)+\Delta\left(t ; U_{2}\right)$. These splits will be repeated until every tree is a collection of even vertex-graphs and/or arc-trees having weight 2 at each vertex. Let $\left\{T_{1}, T_{2}, \ldots, T_{m}\right\}$ be the collection of the trees thus obtained. Then

$$
\Delta(t ; T)=\sum_{i=1}^{m} \Delta\left(t ; T_{i}\right)
$$

Since each $\Delta\left(t ; T_{i}\right)$ is a product of the polynomials of the form $t^{k}-t^{k-1}+$ $\cdots+(-1)^{k}$ or $c(t-1), c>0$, it follows from Proposition 2.1 that each $\Delta\left(-t ; T_{i}\right)$ is trapezoidal. Since deg $\Delta\left(t ; T_{i}\right)=n$ and $\operatorname{sign} \Delta\left(-t ; T_{i}\right)=(-1)^{n}$, it follows that $\Delta_{l}(-t)=\Delta(-t ; T)$ is trapezoidal.

EXAMPLE 6. $\Delta(t)=4 t^{3}-9 t^{2}+9 t-4$
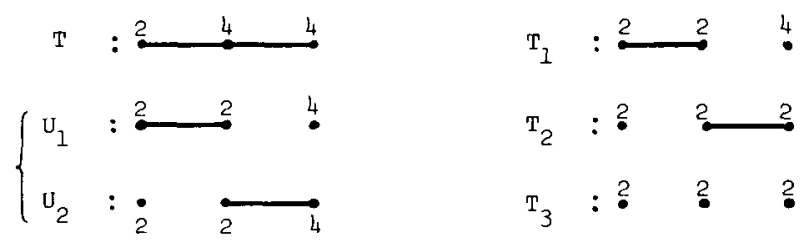

FIGURE 12

Proposition 7.3. Let $l$ be an algebraic link associated with an even excessive positive tree $T$. Then $\Delta_{l}(-t)$ is trapezoidal.

Proof. Split $T$ into a collection of trees, $\left\{T_{1}, T_{2}, \ldots, T_{m}\right\}$ such that each $T_{i}$ is a collection of even excessive positive arc-trees or even vertex-graphs. (Use an 
induction on the number of vertices with valency at least 3.) Since each $\Delta\left(-t ; T_{i}\right)$ is trapezoidal by Proposition 7.1, it follows from (7.1) that $\Delta(-t ; T)$ is trapezoidal and so is $\Delta_{l}(-t)$.

EXAMPLE 7.
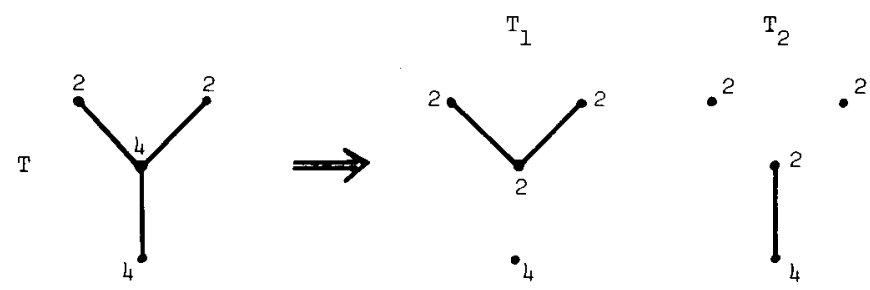

FIGURE 13

\section{Proof of main theorem (II)}

In this section we prove Theorem 5.1 by induction on $k$, the number of components of the uniform decomposition of $T$. We may assume that $T$ is connected.

When $k=1$, Theorem 5.1 is Proposition 7.3, and we are done.

Suppose Theorem 5.1 is true for any tree $T$ which has less than $k$ components in the uniform decomposition. Further we assume inductively that the sign $\Delta(-t ; T)$ is $(-1)^{s}$, where $s$ is the number of positive vertices of $T$. This hypothesis is verified for $k=1$ by Proposition 7.1(3).

Now let $\left\{T_{1}, T_{2}, \ldots, T_{k}\right\}$ be the uniform decomposition of $T$. Then there is a component $T_{i}$ such that the complement $T^{\prime}=\hat{T}\left(T_{i}\right)$ of $T_{i}$ in $T$ is connected. We assume that $T_{k}$ is such a component.

Suppose that $T_{k}$ is a positive tree. (The same proof is valid when $T_{k}$ is negative.)

Let $e_{m}$ be an edge in $A(T)$ which connects a positive vertex $a_{i}$ in $T_{k}$ and a negative vertex $a_{j}$ in $T_{t}$, say. Let $e_{1}, \ldots, e_{p}$ and $e_{m}$ be all edges incident with $a_{j}$. Let $U_{1}$ be a tree obtained from $T$ by removing first $p$ edges $e_{1}, \ldots, e_{p}$ and $w_{1}\left(a_{j}\right)=0$, and $U_{2}$ another tree with $w_{2}\left(a_{j}\right)=w\left(a_{j}\right)$, and apply (6.3) to obtain

$$
\nabla(z ; T)=\nabla\left(z ; U_{1}\right)+\nabla\left(z ; U_{2}\right) .
$$

See Figure 14. 

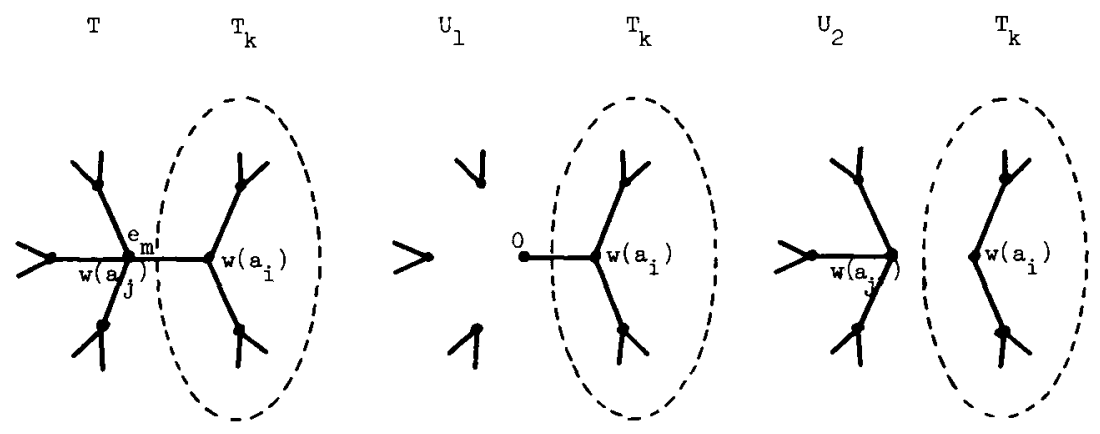

FIGURE 14

Since the algebraic link associated with a subtree $\left\{T_{k}, e_{m}, a_{j}\right\}$ in $U_{1}$ is the (Schubert) product of links associated with the disconnected tree $\hat{T}\left(a_{i}\right)$, we have $\Delta\left(t ; U_{1}\right)=\Delta\left(t ; \hat{T}\left(a_{i}, a_{j}\right)\right)$. On the other hand, by definition,

$\Delta\left(t ; U_{2}\right)=\Delta\left(t ; T_{k}\right) \Delta\left(t ; \hat{T}\left(T_{k}\right)\right)$. By the induction assumption, $\Delta\left(-t ; \hat{T}\left(a_{i}, a_{j}\right)\right)$, $\Delta\left(-t ; T_{k}\right)$ and $\Delta\left(-t ; \hat{T}\left(T_{k}\right)\right)$ are trapezoidal, and hence, $\Delta\left(-t ; U_{1}\right)$ and $\Delta\left(-t ; U_{2}\right)$ are trapezoidal.

Let $n$ be the number of the vertices of $T$ and $s$ the number of the positive vertices of $T$. Then $\operatorname{deg} \nabla\left(z ; U_{1}\right)=n-2$ and $\operatorname{deg} \nabla\left(z ; U_{2}\right)=n$, and also $\operatorname{sign} \Delta\left(-t ; U_{1}\right)=(-1)^{s-1}$ and sign $\Delta\left(-t ; U_{2}\right)=(-1)^{s}$. Therefore, it follows from (8.1) that

$$
\begin{aligned}
\Delta\left(t^{2} ; T\right)= & t^{n} \nabla(t-1 / t ; T)=t^{n} \nabla\left(t-1 / t ; U_{1}\right) \\
& +t^{n} \nabla\left(t-1 / t ; U_{2}\right)=t^{2} \Delta\left(t^{2} ; U_{1}\right)+\Delta\left(t^{2} ; U_{2}\right),
\end{aligned}
$$

and hence, $\Delta(t ; T)=t \Delta\left(t ; U_{1}\right)+\Delta\left(t ; U_{2}\right)$, and we have

$$
\Delta(-t ; T)=(-t) \Delta\left(-t ; U_{1}\right)+\Delta\left(-t ; U_{2}\right) .
$$

Since $(-1)^{s-1} \Delta\left(-t ; U_{1}\right)$ and $(-1)^{s} \Delta\left(-t ; U_{2}\right)$ are positive trapezoidal polynomials, it follows from Proposition 2.1(2) and (8.2) that $(-1)^{s} \Delta(-t ; T)$ is positive and trapezoidal.

The proof of Theorem 5.1 is now completed.

\section{Generalization}

Let $F(n)$ be the orientable surface of Euler characteristic $2-n$ which bounds an oriented torus link of type $(n, 2)$. 


\section{EXAMPLE.}

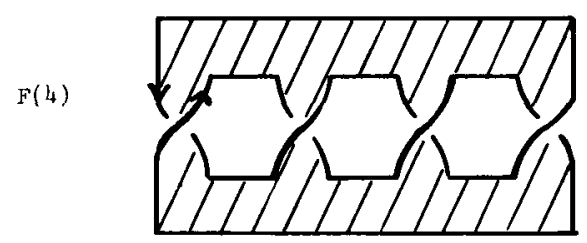

FIGURE 15

It is known [8] that a Seifert surface of an alternating fibre link $l$ is obtained by generalized plumbings (or *-product) from finitely many surfaces $F\left(n_{1}\right), \ldots, F\left(n_{k}\right)$, $n_{i} \neq 0, \pm 1$. A slight modification of the proof of Theorem 5.1 can be applied to a special type of alternating fibre links and we obtain

THEOREM 9.1. Let $l$ be an alternating fibre link. If a Seifert surface of $l$ is obtained by (ordinary) plumbings from $F\left(n_{1}\right), \ldots, F\left(n_{k}\right), n_{i} \neq 0, \pm 1$ in such a way that if $F\left(n_{i}\right)$ and $F\left(n_{j}\right)$ are plumbed then $n_{i} n_{j}<0$, then $\Delta_{l}(-t)$ is trapezoidal.

Figure 16 is an example of such an alternating fibre link.

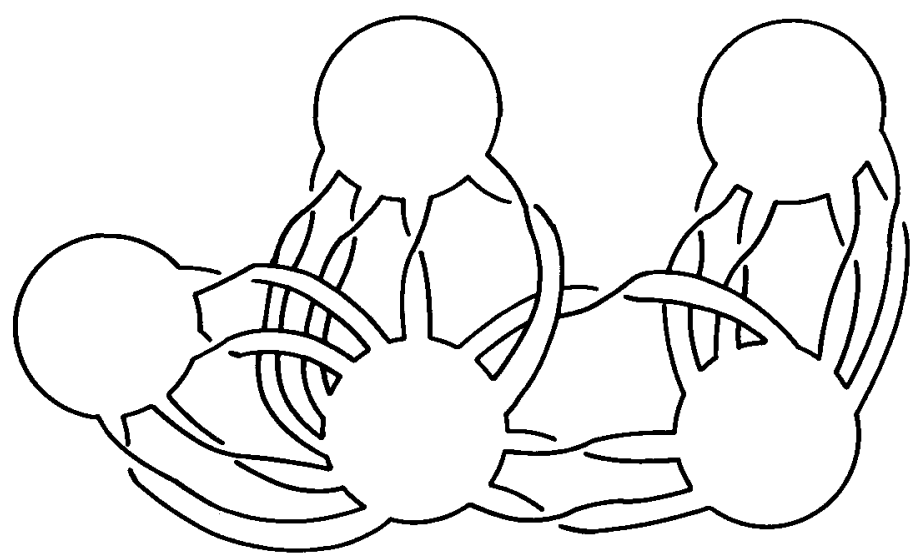

FIGURE 16

\section{References}

[1] C. Bankwitz, 'Uber die Torsionzahlen der alternierenden Knoten', Math. Ann. 103 (1930), $145-161$.

[2] F. Bonahan and L. Siebenmann, 'Algebraic knots', to appear. 
[3] J. H. Conway, 'An enumeration of knots and links, and some of their algebraic properties', pp. 329-358 (Computational Problems in Abstract Algebra, Pergamon Press, New York, 1970).

[4] R. H. Fox, Some problems in knot theory, pp. 168-176 (Topology of 3-manifold and related topics, Ed. M. K. Fort, Prentice-Hall, N. J., 1962).

[5] R. Hartley, 'On two-bridged knot polynomials', J. Austral. Math. Soc. 28 (1979), 241-249.

[6] L. Kauffman, 'The Conway polynomial', Topology 20 (1981), 101-108.

[7] K. Murasugi, 'On the genus of the alternating knot, I, II', J. Math. Soc. Japan 10 (1958), 94-105, 235-248.

[8] K. Murasugi, 'On a certain subgroup of the group of an alternating link', Amer. J. Math. 85 (1963), 544-550.

[9] K. Murasugi, 'On a certain numerical invariant of link types', Trans. Amer. Math. Soc. 117 (1965), 387-422.

[10] R. L. Parris, Pretzel knots (Thesis, Princeton University, 1978).

\section{Department of Mathematics}

University of Toronto

Toronto, Ontario

Canada 